\title{
COSTO-EFECTIVIDAD DE LA KETANSERINA VS. SULFADIAZINA DE PLATA MICRONIZADA EN EL PACIENTE CON PIE DIABÉTICO
}

\section{COST-EFFECTIVENESS OF THE KETANSERIN VS. SULFADIAZINE ON THE PATIENT WITH DIABETIC FOOT}

\author{
Ma. Guadalupe Zúñiga-RamíreZ * \\ Ma. Eugenia Selene Nieto-Álvarez ${ }^{* *}$ \\ LETICIA JiMÉNEZ-RAMÍREZ ${ }^{* * *}$ \\ ENRIQUE VILLARREAL-RÍOs ${ }^{* * * *}$ \\ Lidia MaRTÍNEZ-GONZÁLEZ ${ }^{* * * *}$ \\ LiLiana GaLICIA RODRÍGUEZ ${ }^{* * * * * *}$
}

\begin{abstract}
RESUMEN
Objetivo. Determinar el costo-efectividad de la ketanserina vs sulfadiazina en el paciente con pie diabético. Material y método. Estudio de costo efectividad en pacientes con pie diabético, se integraron dos grupos, los manejados con ketanserina $(n=50)$ y los manejados con sulfadiazina $(n=30)$, se incluyeron a todos los que acudieron al servicio. El costo contempló los insumos para la curación y el medicamento. La efectividad se midió con la reducción de la lesión medida en centímetros y el porcentaje de pacientes curados. Se realizó análisis incremental. Resultados. El costo del centímetro cuadrado de curación en ketanserina es de \$22,43 US y en sulfadiazina $\$ 120,44$ US. La proyección del costo a 5000 pacientes con una lesión de 10 centímetros es $\$ 1.121 .651$ US en ketanserina y \$6.021.787 US en sulfadiazina de plata. Conclusión. En el manejo del pie diabético la relación costo-efectividad de la ketanserina es mejor que la sulfadiazina.
\end{abstract}

Palabras clave: Evaluación de costo-efectividad, pie diabético, ketanserina, sulfadiazina.

\begin{abstract}
Objective. Determine the cost-effectiveness of the ketanserin vs. sulfadiazine on the patient with diabetic foot. Methodology. Study of the cost effectiveness on patients with diabetic foot, there were integrated two groups; the ones managed with ketanserin $(n=50)$, and the ones managed with sulfadiazine $(n=30)$, all that came to the service were included. The cost contemplates the inputs for the cure and the medication. The effectiveness was measured with the reduction of the injury measured in centimeters and the cured patient's percent. Sensitivity and incremental analysis was performed. Results. The cost of square centimeter of healing in ketanserin is $\$ 22.43$ US and in the sulfadiazine $\$ 120.44$ US. The cost of 5000 patients whit an 10 centimeter injury is $\$ 1,121,651$ US

\footnotetext{
* Enfermera, Servicio de Curaciones, Unidad Medica Familiar No 13, Instituto Mexicano del Seguro Social Querétaro, México. E-mail: lic_lupita09@hotmail.com

** Enfermera, Servicio de Curaciones, Unidad Medica Familiar No 13, Instituto Mexicano del Seguro Social Querétaro, México. E-mail: selene.nieto@imss.gob.mx

${ }^{* * *}$ Enfermera, Servicio de Curaciones, Unidad Medica Familiar No 13, Instituto Mexicano del Seguro Social Querétaro, México. E-mail: leticia.jimenez@imss.gob.mx

${ }_{* * * *}$ Médico, Unidad de Investigación Epidemiológica, Instituto Mexicano del Seguro Social Querétaro, México. E-mail: felibree@infosel.net.mx

${ }_{* * * * *}$ Enfermera, Unidad de Investigación Epidemiológica, Instituto Mexicano del Seguro Social Querétaro, México. E-mail: lidia.martinez@imss.gob.mx

${ }^{* * * * * *}$ Lic. en Sistemas, Unidad de Investigación Epidemiológica, Instituto Mexicano del Seguro Social Querétaro, México. E-mail: liliana.galicia@imss.gpb.mx
} 
in ketanserin and $\$ 6,021,787$ US in sulfadiazine. Conclusion. The relation cost-effectiveness of the ketanserin is better than the sulfadiazine one in the management of the diabetic foot.

Key words: Cost-effectiveness evaluation, diabetic foot, ketanserin, sulfadiazine.

Fecha recepción: 13/05/11 Fecha aceptación: 09/04/13

\section{INTRODUCCIÓN}

La diabetes mellitus tipo 2 es una enfermedad crónica degenerativa que se ha constituido como un serio problema de salud pública por la tendencia de la prevalencia y las complicaciones crónicas, entre ellas el pie diabético, alteración clínica inducida por la hiperglucemia sostenida, con componente neuropático, isquémico y traumático (1-6).

Se ha reportado que entre el 10 y el $15 \%$ de los pacientes diabéticos presentan pie diabético al menos una vez en la vida $(7,8)$. En los países desarrollados la prevalencia del pie diabético se encuentra entre el 4 y $10 \%$, con variaciones según el sexo y la edad (7). En México la prevalencia se ha reportado en $10 \%$ y de los pacientes con pie diabéticos el $30 \%$ termina en amputación (7-9).

El esquema de manejo del pie diabético contempla el control metabólico, la antibioticoterapia y la curación de la lesión. En la actualidad la curación se ha acompañado del uso de cicatrizantes o de antibióticos a nivel local con resultados diversos. Entre los más empleados se encuentran la rifampicina, metronidazol, sulfadiazina, italdermol (Tripticum vulgare) y ketanserina (10-14).

En el actual contexto de los servicios de salud caracterizado por los recursos limitados, la evaluación económica y particularmente el análisis costo efectividad han adquirido importancia. La trascendencia de este tipo de estudios radica en la evaluación conjunta entre el costo y el resultado clínico, no obstante es una realidad que en la actualidad este tipo de análisis es poco frecuente en el estudio del paciente con pie diabético
(9), por ello se ha propuesto como objetivo del trabajo la evaluación costo-efectividad de dos alternativas de tratamiento, ketanserina y sulfadiazina de plata micronizada en el paciente con pie diabético $(12,14)$.

\section{MATERIAL Y MÉTODO}

Se realizó un diseño de costo-efectividad en pacientes con diabetes mellitus tipo $2 \mathrm{y}$ lesión de pie diabético que acudieron al servicio de curaciones de la unidad de medicina familiar No 13 del Instituto Mexicano del Seguro Social en el periodo de septiembre de 2009 a agosto de 2010.

Previa autorización se formaron dos grupos, el primero manejado con ketanserina y el segundo con sulfadiazina de plata micronizada. La integración de los grupos fue no aleatoria, a la llegada del paciente al servicio se le explicaron las dos alternativas de manejo y de manera libre eligió una de ellas; con el consentimiento informado el paciente se integró a una de las alternativas de manejo. Para la evaluación de los resultados se adoptó como punto de corte la curación del $100 \%$ de los pacientes del grupo de ketanserina (14 semanas), no obstante el tiempo de tratamiento del grupo de sulfadiazina de plata micronizada se extendió hasta la curación.

En ambos grupos la curación se realizó inicialmente cada tercer día y dependiendo de la evolución la frecuencia varió; el procedimiento incluyó la exposición de la lesión empleando para ello guantes de polietileno; lavado con guante de látex, isodine y gasas; agua bidestilada y gasas para enjuagar; de- 
bridación del tejido necrótico cuando fue necesario; secado del área con gasas estériles; y aplicación con abatelenguas de ketanserina o sulfadiazina. Una vez aplicado el medicamento se cubrió la lesión con gasa estéril y el área periférica a la lesión se lubricó con aceite mineral, finalmente se aplicó vendaje no compresivo del miembro pélvico fijándola con tela adhesiva. Este procedimiento se replicó hasta la remisión de la lesión, momento en el cual el paciente fue egresado.

Se excluyeron aquellos pacientes que no aceptaron el tratamiento y fueron eliminados los que no asistieron a 4 o más curaciones.

Para el cálculo del tamaño de la muestra se utilizó la fórmula de porcentaje para 2 poblaciones con el $95 \%$ de confianza y poder de la prueba del $80 \%$, considerando que el $99 \%$ de los pacientes con ketanserina tenía curación y el $60 \%$ de los pacientes con sulfadiazina de plata tenía curación. Para un total de 20 pacientes por grupo, no obstante se incluyeron a todos aquellos que llegaron en el periodo de estudio, con un total de 50 pacientes del grupo de ketanserina y 30 pacientes del grupo de sulfadiazina.

Se estudió la edad, el sexo y el tiempo de evolución de la diabetes, considerando como tiempo de evolución el número de años transcurridos desde el momento que fue diagnosticado.

* La estimación del costo se realizó en dólares estadounidenses (tipo de cambio $\$ 12,8012$ ), incluyó los costos variables y fijos desde el inicio del tratamiento hasta el egreso.

Costos variables:

- Se identificaron los insumos y la cantidad empleada (gasas, isodine, jabón, abatelenguas, vendas, aceite, guantes, tela adhesiva, ketanserina y sulfadiazina).

- Se identificó el costo unitario de cada insumo.

- La multiplicación de la cantidad de insu- mo por el costo unitario se integró en el costo promedio por insumo.

\section{Costos fijos:}

- Se identificó el sueldo del personal operativo, administrativo, y de servicios básicos, se identificó la inversión en mobiliario, equipo e infraestructura.

- A estos insumos se les calculó el costo por minuto y se relacionó con el total de minutos otorgados a un paciente por atención.

- El resultado se constituyó como el costo fijo promedio.

Costo promedio por alternativa de tratamiento (ketanserina y sulfadiazina de plata): se integró a partir de la suma de los costos variables y fijos.

La efectividad se estableció en función de la reducción semanal de la lesión medida en centímetro cuadrado, así como el porcentaje de población curada.

* El costo efectividad se estableció en relación al costo de cada centímetro cuadrado curado.

El análisis incluyó promedios, porcentajes, intervalos de confianza y análisis incremental.

\section{RESULTADOS}

En el grupo de ketanserina la edad es 59,22 años (IC 95\%; 55,49-62,95), el tiempo de evolución de la diabetes 13,90 años (IC 95\%; $11,60-16,09)$, predominan las mujeres con $52.0 \%$ (IC 95\%; 38,2-65,8), y la superficie del área de la lesión al ingreso es 10,01 cm (IC $95 \% ; 6,22-13,80$ ).

En el grupo de sulfadiazina de plata la edad es 60,56 años (IC 95\%; 55,24-65,88), el tiempo de evolución 17,30 años (IC 95\%; 
14,12-20,47), predomina el sexo masculino $66.7 \%$ (IC 95\%; 49,8-83,6) y la superficie del área al ingreso es $12,68 \mathrm{~cm}$ (IC 95\%; 9,2916,06).

El costo promedio de la opción ketanserina es $\$ 224,55$ US y el de la sulfadiazina de plata $\$ 266,16$ US. En la Tabla 1 se presentan los insumos por grupo, la utilización, el costo unitario y el costo promedio por insumo.

En el grupo de ketanserina el tamaño promedio inicial de la lesión es $10,01 \mathrm{~cm}$, a las 14 semanas el tamaño promedio es $0,00 \mathrm{~cm}$, con $100.0 \%$ de la población con remisión de la lesión; en el grupo de sulfadiazina de plata el promedio inicial de la lesión es 12,68 cm, a las 14 semanas es $10,48 \mathrm{~cm}$, el promedio de modificación (reducción) es 2,21 cm y el porcentaje de población con remisión de la lesión a las 14 semanas es 16,6\%. En la Tabla 2 se presenta por semana el tamaño de la lesión, la modificación y el porcentaje de población con remisión.

El costo por centímetro cuadrado curado en ketanserina es $\$ 22,43$ US y en sulfadiazina de plata \$120,44 US; tratar una lesión de 10 centímetros cuesta $\$ 224,33$ US en el grupo de ketanserina y $\$ 1.204,36$ US en sulfadiazina de plata. En la Tabla 3 se presenta el costo para lesiones de diferente tamaño.

Atender una lesión de pie diabético de 10 centímetros en una población de 5.000 pacientes tiene un costo de $\$ 1.121 .651 \mathrm{US}$ con ketanserina y $\$ 6.021 .787$ US con sulfadiazina de plata. En la Tabla 4 se presenta el análisis incremental para diferentes poblaciones.

Tabla 1. Insumos, utilización, costo unitario y costo promedio para el grupo manejado con ketanserina y el grupo manejado con sulfadiazina de plata.

\begin{tabular}{|c|c|c|c|c|c|}
\hline \multirow[b]{2}{*}{ Insumos } & \multicolumn{2}{|c|}{ Utilización } & \multirow{2}{*}{$\begin{array}{c}\text { Costo } \\
\text { Unitario }\end{array}$} & \multicolumn{2}{|c|}{ Costo promedio } \\
\hline & Ketanserina & $\begin{array}{c}\text { Sulfadiazina } \\
\text { de plata }\end{array}$ & & Ketanserina & $\begin{array}{c}\text { Sulfadiazina } \\
\text { de plata }\end{array}$ \\
\hline Gasas (paquete) & 208,82 & 846,83 & 0,02 & 4,57 & 18,52 \\
\hline Isodine (ml) & 181,60 & $1.369,70$ & 0,001 & 0,14 & 1,07 \\
\hline Agua oxigenada $(\mathrm{ml})$ & 4,84 & - & 0,001 & 0,004 & - \\
\hline Jabón neutro (ml) & 44,66 & 52,77 & 0,001 & 0,03 & 0,04 \\
\hline Abatelenguas (pieza) & 16,52 & 48,07 & 0,01 & 0,09 & 0,26 \\
\hline Vendas (pieza) & 6,78 & - & 0,33 & 2,25 & - \\
\hline Aceite mineral (ml) & 36,80 & 99,57 & 0,003 & 0,11 & 0,31 \\
\hline Guante de polietileno (pieza) & 12,36 & 44,87 & 0,05 & 0,67 & 2,42 \\
\hline Guante de látex (pieza) & 16,56 & 73,13 & 0,09 & 1,44 & 6,34 \\
\hline Tela adhesiva $(\mathrm{cm})$ & 436,52 & $1.367,80$ & 0,04 & 17,05 & 53,42 \\
\hline Ketanserina & 6,78 & - & 17,58 & 119,17 & - \\
\hline Sulfadiazina de plata & - & 396,03 & 0,12 & - & 49,50 \\
\hline Atención (consultas) & 8,04 & 13,66 & 9,83 & 79,03 & 134,27 \\
\hline Costo promedio & & & & 224,55 & 266,16 \\
\hline
\end{tabular}

Los costos se expresan en dólares estadounidenses. 1 US $=12,8012$ pesos mexicanos. 
Tabla 2. Porcentaje de población con remisión, tamaño y modificación de la lesión por semana y tipo de tratamiento.

\begin{tabular}{lcccccc}
\hline & \multicolumn{5}{c}{ Ketanserina } & \multicolumn{3}{c}{ Sulfadiazina de plata } \\
\cline { 2 - 6 } Semana & Tamaño & Modificación & $\begin{array}{c}\text { Porcentaje de } \\
\text { población } \\
\text { con remisión }\end{array}$ & Tamaño & Modificación & $\begin{array}{c}\text { Porcentaje } \\
\text { de población } \\
\text { con remisión }\end{array}$ \\
\hline Inicial & 10,01 & & 12,68 & \\
Primera & 9,41 & 0,6 & 0 & 12,7 & $-0,02$ & 0 \\
Segunda & 7,9 & 2,11 & 8 & 13,85 & $-1,17$ & 0 \\
Tercera & 6,19 & 3,82 & 22 & 11,67 & 1,02 & 0 \\
Cuarta & 5,88 & 4,14 & 32 & 11,5 & 1,18 & 0 \\
Quinta & 4,84 & 5,17 & 36 & 12,4 & 0,28 & 13,3 \\
Sexta & 4,5 & 5,51 & 44 & 11,67 & 1,01 & 13,3 \\
Séptima & 3,48 & 6,53 & 56 & 11,67 & 1,01 & 13,3 \\
Octava & 3,5 & 6,51 & 66 & 11,43 & 1,25 & 13,3 \\
Novena & 3,33 & 6,68 & 70 & 10,99 & 1,69 & 13,3 \\
Décima & 2,3 & 7,71 & 70 & 10,56 & 2,13 & 13,3 \\
Onceava & 1,92 & 8,1 & 78 & 10,92 & 1,76 & 13,3 \\
Doceava & 1,58 & 8,43 & 80 & 10,79 & 1,89 & 13,3 \\
Treceava & 0,72 & 9,29 & 96 & 10,67 & 2,01 & 13,3 \\
Catorceava & 0 & 10,01 & 100 & 10,48 & 2,21 & 16,6 \\
\hline
\end{tabular}

Tabla 3. Costo por centímetros cuadrados curados por tipo de tratamiento.

\begin{tabular}{|c|c|c|c|c|c|c|}
\hline \multirow{3}{*}{ Centímetros } & \multicolumn{3}{|c|}{ Ketanserina } & \multicolumn{3}{|c|}{ Sulfadiazina de plata } \\
\hline & $\begin{array}{l}\text { Centímetros } \\
\text { cuadrados } \\
\text { curados }\end{array}$ & $\begin{array}{c}\text { Costo } \\
\text { promedio }\end{array}$ & $\begin{array}{c}\text { Costo por } \\
\text { centímetro } \\
\text { cuadrado curado }\end{array}$ & $\begin{array}{l}\text { Centímetros } \\
\text { cuadrados } \\
\text { curados }\end{array}$ & $\begin{array}{c}\text { Costo } \\
\text { promedio }\end{array}$ & $\begin{array}{c}\text { Costo por } \\
\text { centímetro } \\
\text { cuadrado curado }\end{array}$ \\
\hline & 10,01 & 224,55 & 22,43 & 2,21 & 266,16 & 120,44 \\
\hline 1 & & & 22,43 & & & 120,44 \\
\hline 2 & & & 44,87 & & & 240,87 \\
\hline 3 & & & 67,30 & & & 361,31 \\
\hline 4 & & & 89,73 & & & 481,74 \\
\hline 5 & & & 112,17 & & & 602,18 \\
\hline 6 & & & 134,60 & & & 722,61 \\
\hline 7 & & & 157,03 & & & 843,05 \\
\hline 8 & & & 179,46 & & & 963,49 \\
\hline 9 & & & 201,90 & & & $1.083,92$ \\
\hline 10 & & & 224,33 & & & $1.204,36$ \\
\hline 11 & & & 246,76 & & & $1.324,79$ \\
\hline 12 & & & 269,20 & & & $1.445,23$ \\
\hline 13 & & & 291,63 & & & $1.565,66$ \\
\hline
\end{tabular}

Los costos se expresan en dólares estadounidenses. 1 US $=12,8012$ pesos mexicanos. 
Tabla 4. Análisis incremental del costo de la curación de una lesión de 10 centímetros, con ketanserina o sulfadiazina de plata en el paciente con lesión de pie diabético.

\begin{tabular}{rrr}
\hline \multirow{2}{*}{ Número de pacientes } & \multicolumn{2}{c}{ Costo } \\
\cline { 2 - 3 } & Ketanserina & Sulfadiazina de plata \\
\cline { 2 - 3 } 100 & 224 & 1.204 \\
500 & 22.433 & 120.436 \\
1.000 & 112.165 & 602.179 \\
5.000 & 224.330 & 1.204 .357 \\
10.000 & 1.121 .651 & 6.021 .786 \\
50.000 & 2.243 .302 & 12.043 .573 \\
100.000 & 11.216 .509 & 60.217 .863 \\
500.000 & 22.433 .018 & 120.435 .726 \\
1.000 .000 & 112.165 .090 & 602.178 .628 \\
& 224.330 .180 & 1.204 .357 .256 \\
\hline
\end{tabular}

\section{DISCUSIÓN Y CONCLUSIÓN}

La importancia de este estudio radica en la evaluación conjunta de los aspectos clínicos y económicos del manejo del pie diabético, patología que limita la capacidad física, social y emocional del paciente, así como el incremento de insumos y costo por parte de la institución $(7,8)$. El resultado marca la pauta para la correcta asignación de recursos y programas institucionales.

Evaluar la efectividad de la intervención a través de la disminución de la lesión pareciera ser el mejor indicador a emplear, lo cual se ve reforzado al tomar como punto de comparación el tiempo de curación $(12,14$, 15). No obstante se debe reconocer como debilidad del trabajo la no aleatorización de los grupos, en este caso podía existir el sesgo de selección de los pacientes.

El costo del medicamento ketanserina es más alto que la sulfadiazina, lo cual podía encarecer el manejo con este esquema, no obstante el costo de la atención por curación en el grupo de sulfadiazina de plata lo compensa y convierte a esta alternativa en la más costosa.

Aunado a ello, con el manejo a base de ketanserina es evidente la mejoría clínica evaluada en función del tamaño y tiempo de remisión de la lesión, así lo señalan los resultados; tener el $50 \%$ de la población con curación de la lesión a las seis semanas habla de una alta efectividad, resultado que al relacionarlo con el costo permite identificar desde la perspectiva costo-efectividad cuál es la mejor alternativa de tratamiento del pie diabético. Lo que muestran los resultados es un retraso en la curación con el esquema de sulfadiazina de plata, la remisión se presenta en la quinta semana y durante nueve semanas más no se presentan nuevos casos, con lo cual se pone en duda la efectividad de este esquema de tratamiento.

El costo por centímetro cuadrado curado es menor con el empleo de ketanserina en una relación de 1 a 5 , esto evidentemente marca una diferencia en el uso del medicamento en el paciente con pie diabético, tanto a nivel personal, al acortar el tiempo de convalecencia, como para el sistema de salud, al permitir el uso más eficiente de los recursos. Ante este panorama, la alternativa de tra- 
tamiento con ketanserina se puede ofrecer como una recomendación a los gestores de los servicios de salud.

Aunado a ello la curación del 100\% de la población en 14 semanas cuando se emplea la ketanserina es una clara muestra del acortamiento del tiempo de evolución que repercutirá en la vida personal del paciente, al reintegrarlo a sus actividades cotidianas (16). En este mismo lapso de tiempo el porcentaje de curación con la sulfadiazina de plata no alcanza ni la quinta parte de la población, con las consecuencias ya señaladas a nivel personal y para el sistema.

La satisfacción del paciente no fue evaluada en este estudio, sin embargo es un punto que se debe considerar en futuras investigaciones ya que forma parte de la calidad de vida del individuo (16); muy probablemente cuando se incluya la satisfacción como parte de la evaluación costo-efectividad, la tendencia será más marcada a la ketanserina.

La identificación del costo por centímetro cuadrado permite hacer un estimado para un caso concreto en función del tamaño de la lesión, aunado a ello ofrece la posibilidad de estimar los insumos en cada caso particular en función de la evaluación clínica (17). Esta información se complementa con el análisis de proyección, escenario en el cual se puede estimar la cantidad requerida de recursos para una población específica.

En conclusión, se puede decir que el manejo del pie diabético tiene una mejor relación costo-efectividad con la ketanserina que con la sulfadiazina de plata micronizada.

\section{AGRADECIMIENTOS}

Los autores agradecen el apoyo del Dr. Sergio Avelino Ramírez López, director de la UMF 13, a la Lic. Martha Rojas Sánchez, jefa de Enfermeras de la UMF 13 y a la Dra. Leticia Martínez Martínez, jefa de Educación e Investigación de la UMF 13.

\section{REFERENCIAS}

1. Watkins P. El ABC de la diabetes. El pie diabético. BMJ.2008; 2: 48-51.

2. Jiménez AM, Riambau V, Escudero JR. Lesiones cutáneas asociadas al pie diabético. En: Lo Roura M, editor. Tratado de Pie Diabético. 2da ed. Madrid: Salvat; 2002. p. 59-69.

3. Contran R, Kuman V, Robbins SI. Patología estructural y funcional. 4ta ed. España: Interamericana; $1990.1048-58$ p.

4. Linden E, Cai W, He JC, Xue C, Li Z, et al. Endothelial dysfunction in patients with chronic kidney disease results from advanced glycation end products (AGE)Mediated Inhibition of endothelial nitric oxide synthase through RAGE Activation. Clin J Am Soc Nephrol. 2008; 3(3): 691-98.

5. Galkowska H, Wojewodzka U, Olszewski WL. Chemokines, cytokines and growth factors in keratinocytes and dermal endothelial cells in the margin if chronic diabetic foot ulcers. Wound Repair Regen. 2006; 14(5): 558-65.

6. Bennett NT, Allien GS. Growth factors and wound healing: Part II. Role in normal and chronic wound healing. Am J Surg. 1993; 166(1): 74-81.

7. Secretaría de Gobernación. Encuesta Nacional de Salud y Nutrición 2009 [Internet]. México; [citado 17 enero 2011]. Disponible en: htp://www.dof.gob.mx/ documental 3868/salud/htm.

8. Sistema de información de atención integral de la salud, SIAIS. Unidad de Medicina Familiar No 13, Dirección de Prestaciones Médicas Querétaro México. Instituto Mexicano del Seguro Social, 2009-2010.

9. Doherty G. Diagnóstico y tratamiento quirúrgicos. Amputación. 9 ed. Colombia: Manual Moderno; 2009. 857-61 p.

10. SANOFI-AVENTIS. Rifocina [Internet]. Paraguay: Vademécum; 2011 [ci- 
tado 3 enero 2011]. Disponible en: http://py.prvademecum.com/producto. php? producto $=179$

11. Mensa J, Gatell JM, Azanza JR, Domínguez-Gil A et al. Guía de terapéutica antimicrobiana. 18 ed. Barcelona: Elsevier Doyma; 2008.

12. Instituto Mexicano del Seguro Social [Internet]. México DF: Instituto Mexicano del Seguro Social; [2011]. Cuadro Básico Medicamentos; [citado 25 enero 2011]. Disponible en: htp://www.imss.gob.mx/ transparencia/cuadrosbasicos/Documentos/CBM.pdf

13. Herruzo-Cabrera R, García-Torres V, Vizcaíno-Alcaide MJ. Evaluation of the penetration strength, bactericide efficacy and spectrum of action of several antimi- crobial creams again is isolated microorganisms is a burn center. Burns. 1992; 18(1): 39-44.

14. Jonasson J, Ye W, Sparén P, Apelqvist J, Nyrén $\mathrm{O}$ et al. Risks of nontraumatic lower-extremity amputations in patients with type 1 diabetes. Diabetes Care. 2008; 31(8): 1536-540.

15. Edmon M, Forester A. El ABC de la cicatrización de las heridas. BMJ. 2008; 2: 56-60.

16. Lerman I. Atención integral del paciente diabético. 2da ed. Madrid: McGraw-Hill; 2001. p. 205-13.

17. Raparaz-Asensio L, Sánchez-García C. El pie diabético. An Med Interna. 2004; 21(9): 417-19. 